\title{
The AGILE observations of the hard and bright GRB 100724B
}

E. Del Monte ${ }^{1}$, G. Barbiellini ${ }^{2,3}$, I. Donnarumma ${ }^{1}$, F. Fuschino ${ }^{4}$, A. Giuliani ${ }^{5}$, F. Longo ${ }^{2,3}$, M. Marisaldi ${ }^{4}$, G. Pucella ${ }^{6}$, M. Tavani ${ }^{1,7}$, M. Trifoglio ${ }^{4}$, A. Trois ${ }^{1}$, A. Argan ${ }^{1}$, A. Bulgarelli ${ }^{4}$, P. Caraveo ${ }^{5}$, P. W. Cattaneo ${ }^{8}$, A. W. Chen ${ }^{5}$, E. Costa $^{1}$, F. D’Ammando ${ }^{9}$, G. Di Cocco ${ }^{4}$, Y. Evangelista ${ }^{1}$, M. Feroci ${ }^{1}$, M. Galli ${ }^{10}$, F. Gianotti ${ }^{4}$, C. Labanti ${ }^{4}$, I. Lapshov ${ }^{1}$, F. Lazzarotto ${ }^{1}$, P. Lipari ${ }^{11}$, S. Mereghetti ${ }^{5}$, E. Moretti ${ }^{2,3}$, A. Morselli ${ }^{7}$, L. Pacciani ${ }^{1}$, A. Pellizzoni ${ }^{12}$, F. Perotti ${ }^{5}$, G. Piano ${ }^{1}$, P. Picozza ${ }^{7}$, M. Pilia ${ }^{12,13}$, M. Prest ${ }^{13}$, M. Rapisarda ${ }^{6}$, A. Rappoldi ${ }^{8}$, S. Sabatini ${ }^{1}$, P. Soffitta ${ }^{1}$, E. Striani ${ }^{1}$, E. Vallazza ${ }^{2}$, S. Vercellone ${ }^{9}$, V. Vittorini ${ }^{1}$, L. A. Antonelli ${ }^{14,15}$, S. Cutini ${ }^{14,16}$, C. Pittori ${ }^{14,16}$, P. Santolamazza ${ }^{14,16}$, F. Verrecchia ${ }^{14,16}$, P. Giommi ${ }^{14,17}$, and L. Salotti ${ }^{17}$

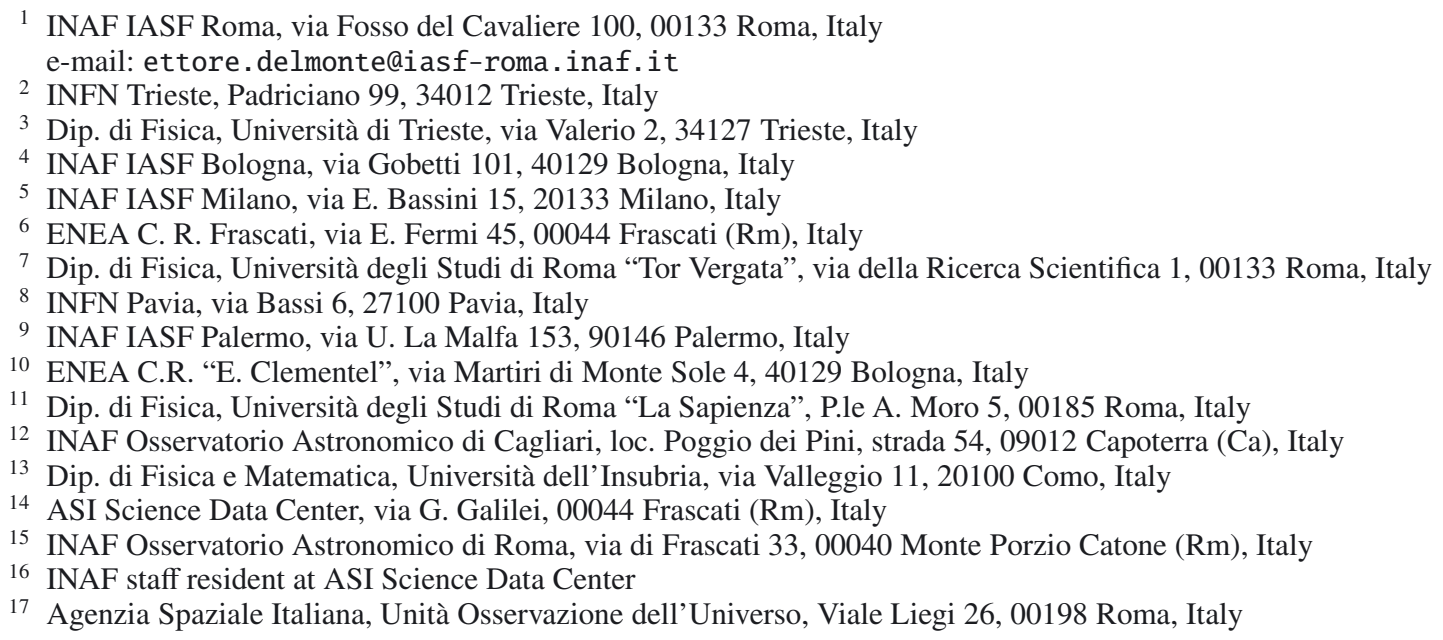

Received 8 April 2011 / Accepted 8 September 2011

\section{ABSTRACT}

\begin{abstract}
The observation of gamma ray bursts (GRBs) in the gamma ray band has been advanced by the AGILE and Fermi satellites after the era of the Compton Gamma-Ray Observatory. AGILE and Fermi are showing that the GeV-bright GRBs share a set of common features, particularly the high fluence from the $\mathrm{keV}$ up to the $\mathrm{GeV}$ energy bands, the high value of the minimum Lorentz factor, an extended emission of gamma rays, which is often delayed with respect to lower energies, and finally the possible multiple spectral components. GRB 100724B, localised in a joint effort by Fermi and the InterPlanetary Newtork, is the brightest burst detected in gamma rays so far by AGILE. Characteristic features of GRB 100724B are the simultaneous emissions at $\mathrm{MeV}$ and $\mathrm{GeV}$, without delayed onset or any time lag as shown by the analysis of the cross correlation function, and the significant spectral evolution in hard $\mathrm{X}$-rays over the event duration. In this paper we show the analysis of the AGILE data of GRB 100724B and discuss its features in the context of the bursts observed so far in gamma rays and the recently proposed models.
\end{abstract}

Key words. gamma rays: general - gamma-ray burst: individual: GRB 100724B

\section{Introduction}

In more than thirty years of study a wealth of information has been gathered about the prompt emission of gamma ray bursts (GRBs) in hard X-rays. The interested reader may find recent and comprehensive reviews by Piran (2004), Mészáros (2006), and Gehrels et al. (2009), among others. In comparison, before the launch of the AGILE (Tavani et al. 2009) and Fermi (Michelson et al. 2010) satellites, limited information was available about the GRB higher energy component. In fact, the EGRET spark chamber (Kanbach et al. 1988) aboard the satellite-borne Compton Gamma-Ray Observatory only detected a small number of GRBs in the energy band between hundreds of $\mathrm{MeV}$ and few GeV: GRB 910503 (Schneid et al. 1992), GRB 910601 (Dingus 1995), GRB 910814 (Schaefer et al. 1998), GRB 920622 (Schneid et al. 1995), GRB 930131 (Kouveliotou et al. 1994; Sommer et al. 1994), GRB 940217 (Hurley et al. 1994), and GRB 940301 (Schneid et al. 1995; Dingus 1995). Combined spectra of a sample of 15 GRBs detected by the Compton Gamma-Ray Observatory are analysed by Kaneko et al. (2008) in an energy range between tens of keV (from BATSE, Fishman et al. 1992) and tens of MeV (from the EGRET TASC calorimeter). EGRET also discovered that, compared with the $\mathrm{keV}$ and $\mathrm{MeV}$ energy bands, the gamma ray emission may last much longer, with the exceptional case of GRB 940217 (Hurley et al. 1994), whose gamma ray component 
lasted for $\simeq 5400 \mathrm{~s}$ and from which a photon of $18 \mathrm{GeV}$ was detected $\simeq 5000 \mathrm{~s}$ after the trigger.

In recent years, after the demise of the Compton GammaRay Observatory, the observation of GRBs in the $\mathrm{MeV}$ to $\mathrm{GeV}$ band is continuing thanks to the gamma ray imagers aboard the AGILE and Fermi satellites. GRBs observed in gamma rays by AGILE and Fermi, which include both long and short events, exhibit peculiar and distinctive features. The gamma ray emission generally starts during the prompt phase and may be simultaneous with the hard X-ray component, as in GRB 090217A (Ackermann et al. 2010a), or may show a delay, with the most striking case being the short GRB 090510 (Giuliani et al. 2010; Ackermann et al. 2010b; Kumar \& Barniol Duran 2010). Moreover, in the majority of GRBs observed so far in gamma rays, the $\mathrm{GeV}$ component has a longer duration than the $\mathrm{keV}-\mathrm{MeV}$ one. A recent and detailed review of the GRB features in gamma rays is provided, for example, by Zhang (2011).

The Fermi observation of GRB 090902B (Abdo et al. 2009a) is a paradigmatic example of detecting different components in the spectrum of a gamma ray bright GRB. We recall here that the GRB spectral energy distribution can be modelled using the phenomenological Band function (Band et al. 1993), and on average the parameters show a peak energy around $\simeq 300 \mathrm{keV}$ and photon indices $\simeq-1.1$ and $\simeq-2.3$ for the low and high energy powerlaws, respectively (Preece et al. 2000). The spectrum of GRB 090902B can be modelled using two components: the Band function in the energy band between $50 \mathrm{keV}$ and $40 \mathrm{MeV}$ and an additional powerlaw, fitting simultaneously the emission below $50 \mathrm{keV}$ and above $100 \mathrm{MeV}$. In other events, such as GRB 080514B observed by AGILE (Giuliani et al. 2008) and GRB 080916C studied by Fermi (Abdo et al. 2009b), the spectral shape is the same from $\mathrm{keV}$ up to $\mathrm{GeV}$ energies, and a single Band function is an adequate model for the whole spectrum.

Some hints that the high fluence is an inherent peculiarity of the GeV-emitting GRBs date back to the EGRET era. For example, GRB 940217 has a remarkable fluence of $(6.6 \pm 2.7) \times$ $10^{-4} \mathrm{erg} \mathrm{cm}^{-2}$ above $20 \mathrm{keV}$ (Hurley et al. 1994). From the analysis of a sample of twelve GRBs detected in the $\mathrm{GeV}$ band by the Fermi Large Area Telescope (LAT), Ghisellini et al. (2010) find that a common property is their high fluence measured in the $8 \mathrm{keV}-10 \mathrm{MeV}$ energy band by the Gamma-ray Burst Monitor (GBM), belonging to the $2 \sigma(95 \%)$ to $3 \sigma(99.7 \%)$ highest tail in the distribution from the catalogue of $121 \mathrm{GRBs}$ of the latter instrument (until October 2009).

Another important characteristic of GRBs detected in gamma rays is the remarkably high value of their bulk Lorentz factor. Since a spectral cutoff is not detected in the GeV interval, the optical depth of the interactions of gamma rays on lowenergy photons is small (Baring \& Harding 1997; Lithwick \& Sari 2001). The minimum bulk Lorentz factor can consequently be estimated based on the maximum detected energy and the time variability, on the hypothesis that the photon number is distributed following a powerlaw, as reported on the supporting online material of the paper by Abdo et al. (2009b). Using this method, minimum Lorentz factor values ranging from hundreds up to about one thousand (for GRB 090510, Ackermann et al. 2010b) are derived for gamma ray bright GRBs. For comparison, using a similar method (discussed by Lithwick \& Sari 2001), Rossi et al. (2011) estimate a minimum of a few hundreds on the initial bulk Lorentz factor of the non-GeV emitting GRB 080928, detected up to $150 \mathrm{keV}$ by Fermi and Swift (Gehrels et al. 2004). However, the estimation of the minimum Lorentz factor depends on the model of the photon density in the burst emission mechanism and values lower by a factor of two to three are found, for example, by Hascoët et al. (2011).

The first detections of $\mathrm{GeV}$ photons from GRBs by AGILE and Fermi have raised a debate about the emission mechanisms. A complete and exhaustive review of all the available models is far beyond the scope of this paper. In the following, we concentrate mainly on the dichotomy between internal shock (i.e. prompt emission) and external shock (i.e. afterglow emission) scenarios to explain the peculiar characteristics of the GRB gamma ray component, especially the delayed onset and the longer duration with respect to lower energies, the overall spectral shape and the possible presence of additional spectral components. Ghisellini et al. (2010) discuss the properties of twelve GRBs detected by Fermi and interpret the gamma ray emission as afterglow, particularly on the basis of the temporal decay slope, similar to that of afterglows observed in X-rays. Following their interpretation, the decay slope is also consistent with a radiative regime, in which all the dissipated energy is radiated away. The gamma ray emission is also interpreted as afterglow by Kumar \& Barniol Duran (2010), who establish a correlation between the gamma ray flux (above $100 \mathrm{MeV}$ ) and the flux of the X-ray and optical afterglows at a late time. From the synchrotron emission model, the authors can estimate the properties of the external shock emission, such as the fraction of energy in electrons and magnetic field, the circumstellar medium density, and the burst kinetic energy, consistently in both the prompt flux above $100 \mathrm{MeV}$ and the late-time X-ray and optical afterglows. The external shock scenario is challenged, for example, by Maxham et al. (2011) based on the fact that, above $100 \mathrm{MeV}$, the external shock model that fits the data at a late time (50-500 s after trigger) gives just a fraction of the flux at an early time (1-50 s after trigger). The authors consequently interpret the gamma ray flux as mainly due to the internal shock, with only a small contribution from the external shock.

The paper is organised as follows: the detection of GRB 100724B is described in Sect. 2, the analysis of the AGILE data is reported in Sect. 3, and finally we discuss our results and draw our conclusions in Sect. 4.

\section{Detection of GRB 100724B}

GRB 100724B was localised by the GBM (Meegan et al. 2009) aboard Fermi on 24 July 2010 00:42:05.98 UT at the position $\mathrm{RA}=124.16^{\circ}, \mathrm{Dec}=74.42^{\circ}$ with a statistical error of $1.0^{\circ}$ and $\mathrm{a}$ systematic error in the range between $2^{\circ}$ and $3^{\circ}$ (Bhat 2010). The Fermi-LAT (Atwood et al. 2009) localised the GRB at a position $\left(\mathrm{RA}=120.04^{\circ}, \mathrm{Dec}=76.74^{\circ}\right)$ consistent with GBM and with an error box of $1.1^{\circ}$ (Tanaka et al. 2010). The position with the smallest error region is a strip in the sky with $1.2^{\circ}$ length and $0.2^{\circ}$ width centred on $\mathrm{RA}=118.8^{\circ}, \mathrm{Dec}=75.8^{\circ}$ (Guiriec et al. 2011), which is obtained from the intersection of the LAT error box at $90 \%$ confidence level and the annulus produced by the InterPlanetary Network (IPN) with the GBM, Konus-Wind, and MESSENGER data.

GRB 100724B also triggered (Marisaldi et al. 2010) the AGILE Minicalorimeter (MCAL; Labanti et al. 2009) on 24 July 2010 00:42:00 UT, hereafter considered as the event trigger time $\left(t_{0}\right)$. The burst was also detected (Giuliani 2010) by the AGILE Gamma Ray Imaging Detector (GRID; Prest et al. 2003) with a dedicated analysis aimed at finding the counterpart of GRBs localised in hard X-rays by other instruments (see Moretti et al. 2009, for a description of the technique). At the trigger time only some of the AGILE contacts with the Malindi ground station were available for the telemetry downlink, consequently the 
hard X-ray imager SuperAGILE (see Feroci et al. 2007, for a description) was configured not to collect data in order to ensure the complete telemetry storage by the GRID.

At the time of the GBM detection, the GRB distance from the Sun was only $54.5^{\circ}$ and the Swift satellite could not perform follow-up observations owing to Sun aspect constraints. The GRB position went out from the Swift Sun aspect constraints on 2 August 2010 and two follow-up observations were performed on slightly different positions, starting $8.41 \times 10^{5} \mathrm{~s}$ (with observation id 20144) and $8.47 \times 10^{5} \mathrm{~s}$ (with observation id 20145) after trigger respectively. No afterglow candidates are found in the two pointings and no more follow-up observation were performed due to the tight schedule of the Swift mission, thus GRB 100724B lacks an associated afterglow and a redshift.

\section{The AGILE observation of the prompt emission}

\subsection{Data reduction}

The MCAL data are stored in photon-by-photon mode in a temporary buffer and are provided to telemetry only on trigger (see Fuschino et al. 2008, for a description). In the case of GRB $100724 \mathrm{~B}$, the buffer contains data from $\simeq 40$ s before until $\simeq 200 \mathrm{~s}$ after trigger. We did not detect any GRB precursor, at a significance level of $5 \sigma$, in the available MCAL data before trigger, thus all these events are used to estimate the instrument background. We selected the MCAL data above $0.3 \mathrm{MeV}$ energy, thus ensuring a negligible contamination from the instrumental electronic noise.

Since January 2010, AGILE is operating in a spinning mode with an angular velocity of $\simeq 0.8^{\circ}$ per second around the axis pointed toward the Sun. The spinning mode produces the apparent motion of the sources in the GRID field of view (FoV) and the "transit" duration depends on the source position and the satellite attitude. In the case of GRB 100724B, the distance between the source position and the satellite boresight is $\simeq 65^{\circ}$ at the trigger time, decreases down to a minimum value of $\simeq 35^{\circ}$ at $t_{0}+60 \mathrm{~s}$, and then increases again up to $\simeq 70^{\circ}$ at $t_{0}+140 \mathrm{~s}$. Assuming an FoV of $60^{\circ}$ radius for the GRID, GRB 100724B is contained within the FoV between $t_{0}+6 \mathrm{~s}$ and $t_{0}+125 \mathrm{~s}$ (thus the measured duration is $119 \mathrm{~s}$ ) and then again between $t_{0}+410 \mathrm{~s}$ and $t_{0}+529 \mathrm{~s}$.

We selected the GRID data of GRB 100724B in the time interval defined above (from $t_{0}+6 \mathrm{~s}$ until $t_{0}+125 \mathrm{~s}$ ) and in a $12^{\circ}$ radius from the source position. To exclude the contamination from the Earth albedo, we selected photons with an angular distance greater than $90^{\circ}$ from the Earth centre. For GRB 100724B we classified the events in the photon list with the F4 filter (Giuliani et al. 2008, and references therein), which provides the highest statistic. Using these cuts and selections we extracted 57 events from GRB 100724B with reconstructed energy from $22 \mathrm{MeV}$ to $3.5 \mathrm{GeV}$ (see Fig. 1). The GRB is localised by GRID at $\mathrm{RA}=124.3^{\circ}, \mathrm{Dec}=78.5^{\circ}$ with a $95 \%$ confidence level uncertainty of $1.5^{\circ}$ radius (including a systematic contribution of $0.1^{\circ}$ ), at $3.0^{\circ}$ from the centre of the IPN error region (reported by Guiriec et al. 2011).

\subsection{Lightcurves and cross-correlation}

The statistics of the MCAL data allowed us to extract the lightcurves in three energy bands: $0.3-1 \mathrm{MeV}, 1-5 \mathrm{MeV}$, and above $5 \mathrm{MeV}$. We accumulated the GRID lightcurve by converting the detected counts into flux using the instrument's effective area as a function of the off-axis angle, depending on
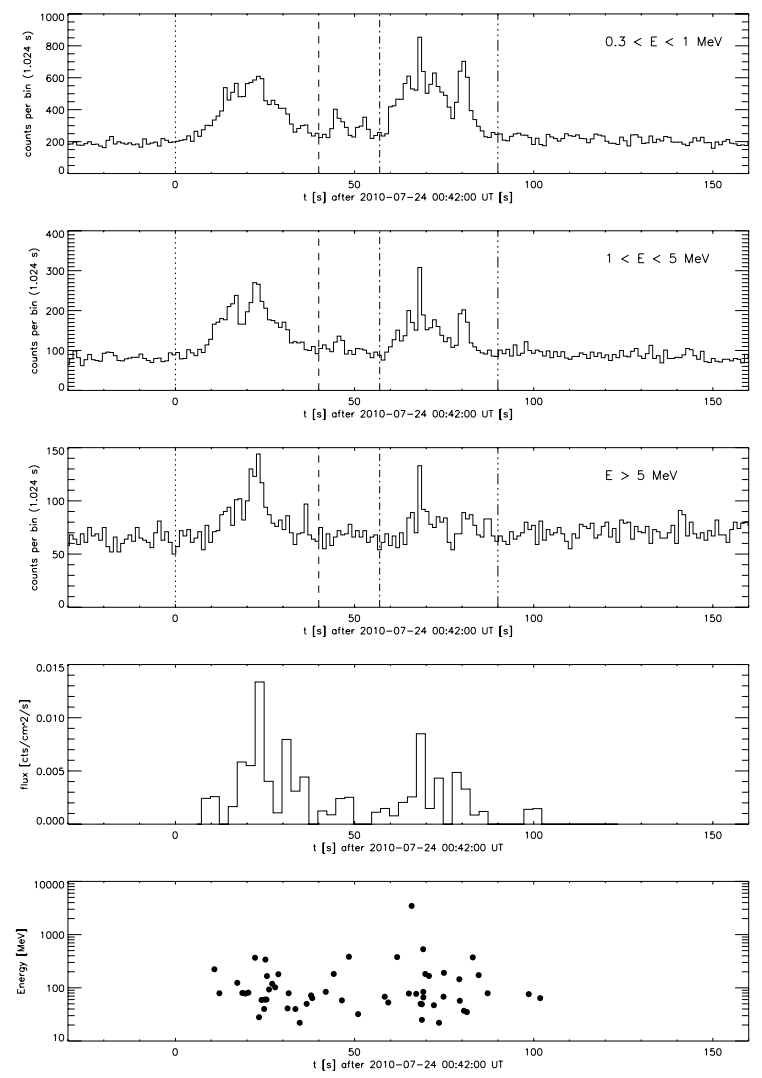

Fig. 1. Lightcurves of GRB 100724B: from the top down MCAL 0.3-1 MeV, MCAL 1-5 MeV, MCAL above $5 \mathrm{MeV}$, GRID flux corrected for the effective area and GRID scatter plot of energy vs. time.

time. The superposition of the MCAL and GRID lightcurves of GRB 100724B is shown in Fig. 1.

GRB $100724 B$ is significantly detected by MCAL up to $\simeq 80 \mathrm{MeV}$ energy. We searched backward for possible precursors in the MCAL available data, until $t_{0}-40 \mathrm{~s}$, but we did not find any at a significance level of $5 \sigma$. The time intervals $T_{90}$ and $T_{50}$ enclose the burst counts from $5 \%$ to $95 \%$, and $25 \%$ to $75 \%$ of the total, respectively, and are calculated using the standard procedure outlined by Koshut et al. (1996). From the counts over the full MCAL energy range $(350 \mathrm{keV}-100 \mathrm{MeV}), T_{50}=50.6 \pm 1.1 \mathrm{~s}$ and $T_{90}=96.4 \pm 10.8 \mathrm{~s}$. Our $T_{90}$ is shorter, but still compatible, with the $111.6 \mathrm{~s}$ value reported by GBM (Bhat 2010) for the 50-300 keV energy band. It is important to point out here that the MCAL response, hence the measure of the burst duration in the MeV band, is negligibly affected by the AGILE spinning mode.

We studied the shape of the MCAL lightcurve by selecting the data in the three energy ranges listed above $(0.3-1 \mathrm{MeV}$, $1-5 \mathrm{MeV}$, and above $5 \mathrm{MeV})$. The lightcurve between $t_{0}$ and $t_{0}+96.4 \mathrm{~s}$ is composed of two bumps, both detected in the whole energy range and characterised by internal structure, interleaved by an interbump pause region, where two softer and fainter peaks are present, the first one only visible below $5 \mathrm{MeV}$ and the second one only below $1 \mathrm{MeV}$ (see Fig. 1). The time series in the three energy bands are qualitatively similar, with the two main bumps peaking at similar values of time. Above $5 \mathrm{MeV}$ most of the internal structure in the bumps is not statistically significant above the background, for example the narrow peak at the end of the second bump (at $\simeq t_{0}+80 \mathrm{~s}$ ), while a narrow peak is present only above $5 \mathrm{MeV}$ around $\simeq t_{0}+35 \mathrm{~s}$ but with a significance of only $3 \sigma$. 

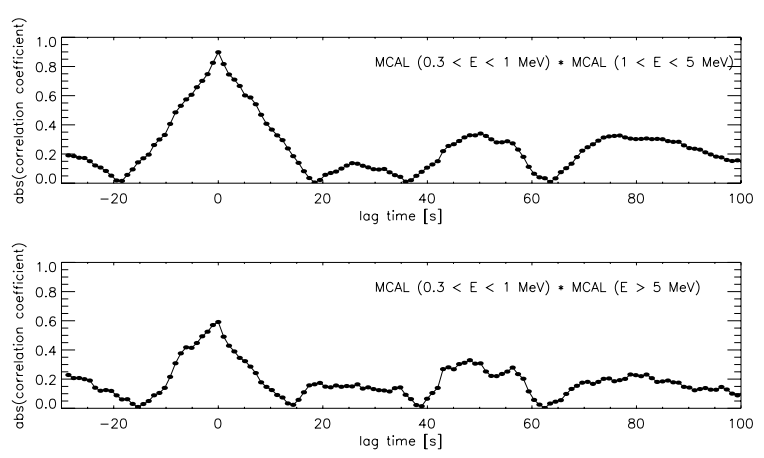

Fig. 2. Cross-correlation of GRB 100724B between the MCAL lightcurve at $0.3-1 \mathrm{MeV}$ and the lightcurves from 1 to $5 \mathrm{MeV}$ (top panel) and above $5 \mathrm{MeV}$ (bottom panel).

We studied the presence of energy-dependent lags in GRB 100724B by computing the cross-correlation function (CCF) of the time series in different energy ranges. The CCF is defined in most textbooks of statistical analysis (see for example Bevington \& Robinson 2003). In Fig. 2 we show the absolute value of the CCF between the MCAL lightcurve in the $0.3-1 \mathrm{MeV}$ energy band and the lightcurves in $1-5 \mathrm{MeV}$ (top panel) and above $5 \mathrm{MeV}$ (bottom panel). We use a time lag between $-30 \mathrm{~s}$ and $+160 \mathrm{~s}$ and a bin size of $1.024 \mathrm{~s}$ (the same as in Fig. 1), the shortest allowed by the statistics of the data. The peak in the CCF is taken as the measure of spectral lag (Norris 2002). We can see from Fig. 2 that the time lag in the MCAL lightcurves is less than $1.024 \mathrm{~s}$.

The position of the maximum emission in the first bump does not change significantly in the three energy bands, as demonstrated with the CCF. Conversely, the first bump width shows variations as a function of energy, measured by assuming a symmetric Gaussian shape. From the Gaussian fit we found, again, that the position does not change significantly with energy, while the width, consistent in the first and second bands, is smaller in the highest energy interval. The Gaussian function is just a qualitative model for the bump shape, and the high values of the reduced chi square, which are not formally acceptable, are dominated by the internal peak structure. Similarly, the position of the maximum emission in the second main bump does not change significantly in the three energy bands but, in this case, the bump shape is dominated by the superposition of many overlapping peaks and thus cannot be modelled using the same method as applied to the first bump.

The GRID lightcurve shows two broad bumps, at the same position as in MCAL, without evident features in the interbump region (see Fig. 1). The average background level in the GRID time series around the time interval of GRB 100724B is $0.12 \mathrm{cts} \mathrm{s}^{-1}$, corresponding to a flux of $4 \times 10^{-4} \mathrm{ph} \mathrm{cm}^{-2} \mathrm{~s}^{-1}$ when corrected for the effective area at $100 \mathrm{MeV}$ for an average off-axis angle of $50^{\circ}$.

The onset of the gamma ray emission does not show any significant delays with respect to the hard X-ray band; in fact the first photon is detected by GRID at $t_{0}+10.9 \mathrm{~s}$, during the rise of the first bump in MCAL, and has an energy of $\simeq 220 \mathrm{MeV}$. The highest energy photon $(\simeq 3.5 \mathrm{GeV})$ is detected by GRID immediately before the maximum of the second bump in the MCAL lightcurve. The narrow peak around $\simeq t_{0}+35 \mathrm{~s}$ in the MCAL lightcurve above $5 \mathrm{MeV}$ is also evident in the GRID data.

The absolute value of the CCF between the time series of the GRID and MCAL (without background subtraction) in the three energy bands (0.3-1 MeV, $1-5 \mathrm{MeV}$, and above $5 \mathrm{MeV}$ )
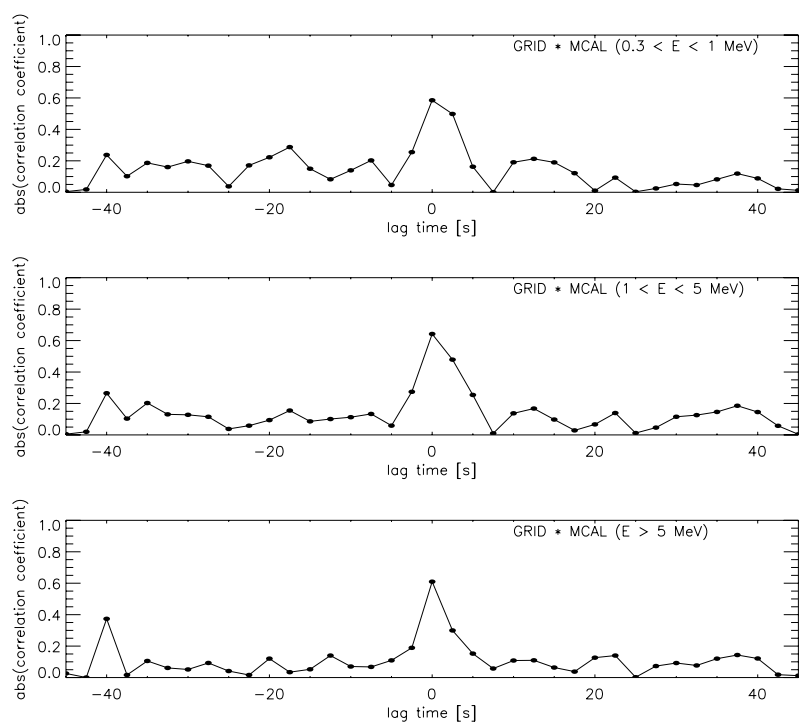

Fig. 3. Cross-correlation of GRB 100724B between the lightcurves of the GRID and of the MCAL between 0.3 and $1 \mathrm{MeV}$ (top panel), between 1 and $5 \mathrm{MeV}$ (middle panel) and above $5 \mathrm{MeV}$ (bottom panel).

is shown in Fig. 3. In the cross-correlation we used for all the lightcurves the same time bin of $2.5 \mathrm{~s}$, which is the shortest value allowed by the statistics of the GRID data and the same one as used for the lightcurve in Fig. 1. Since the GRB is within the GRID FoV only from $t_{0}+6 \mathrm{~s}$ until $t_{0}+125 \mathrm{~s}$, we used a time lag range between $-45 \mathrm{~s}$ and $+45 \mathrm{~s}$, which is different from the $\mathrm{CCF}$ at lower energy (in Fig. 2), in which data from a wider time interval can be used. Again, the correlation coefficient is maximum at a zero time lag, indicating the absence of a spectral lag longer than $2.5 \mathrm{~s}$ between the time series of GRID and MCAL. This result agrees with the comparison between the MCAL and GRID lightcurves, indicating that the position of the maximum emission is coincident in the first bump at $t_{0}+22.5 \mathrm{~s}$, as well as in the second one at $t_{0}+67.5 \mathrm{~s}$ (see Fig. 1). The width of the first bump in GRID is similar to MCAL ( $\simeq 10 \mathrm{~s}$ at half maximum), while the width of the second one can only be estimated with difficulty because of the low-quality statistics.

Recent observations of GeV-bright GRBs have shown that gamma rays are detected in an extended emission prompt phase, lasting well after $T_{90}$ and up to tens or even hundreds of seconds after trigger. The prototype of this behaviour is represented by GRB 940217 (Hurley et al. 1994), and recent cases are, for example, GRB 080514B (Giuliani et al. 2008) detected by AGILE, GRB 090510 (Giuliani et al. 2010; Ackermann et al. 2010b; Kumar \& Barniol Duran 2010) studied by AGILE and Fermi, and GRB 080825C (Abdo et al. 2009c) observed by Fermi. The study of this phenomenology in GRB 100724B is complicated by AGILE operating in a spinning mode, consequently the burst "exits" from the GRID FoV on $t_{0}+125 \mathrm{~s}$, and the subsequent "transit" lasts between $t_{0}+410 \mathrm{~s}$ and $t_{0}+529 \mathrm{~s}$. We extracted the GRID data in this second time interval, using the same cuts and selections of the first interval (represented in Fig. 1), and we found only 11 events. When taking the same background level measured before trigger into account, thanks to the stability of the GRID particle background in the equatorial low Earth orbit (Tavani et al. 2009), the signal-to-noise ratio in this case is just $1.0 \sigma$. The significance level remains similar if we reduce the extraction radius down to $10^{\circ}$, obtaining $1.4 \sigma$, or even $8^{\circ}$, resulting in $1.5 \sigma$. 
Table 1. Fit results of the MCAL spectra of GRB 100724B, with times relative to the trigger time $t_{0}$ corresponding to 2010-07-24 00:42:00 UT.

\begin{tabular}{lccc}
\hline \hline Time interval & Photon index & $\chi_{r}^{2}$ (d.o.f.) & $\begin{array}{c}\text { Flux } 0.5-100 \mathrm{MeV} \\
{\left[\mathrm{erg} \mathrm{cm}^{-2} \mathrm{~s}^{-1}\right]}\end{array}$ \\
\hline whole $\left(t_{0}, t_{0}+90 \mathrm{~s}\right)$ & $2.13_{-0.04}^{+0.05}$ & $0.923(61)$ & $2.38 \times 10^{-6}$ \\
$\mathrm{~A}\left(t_{0}, t_{0}+40 \mathrm{~s}\right)$ & $2.01 \pm 0.04$ & $1.226(54)$ & $3.35 \times 10^{-6}$ \\
$\mathrm{~B}\left(t_{0}+40 \mathrm{~s}, t_{0}+57 \mathrm{~s}\right)$ & $2.19_{-0.19}^{+0.26}$ & $0.661(21)$ & $9.18 \times 10^{-7}$ \\
$\mathrm{C}\left(t_{0}+57 \mathrm{~s}, t_{0}+90 \mathrm{~s}\right)$ & $2.35_{-0.07}^{+0.08}$ & $0.663(42)$ & $2.24 \times 10^{-6}$ \\
\hline
\end{tabular}

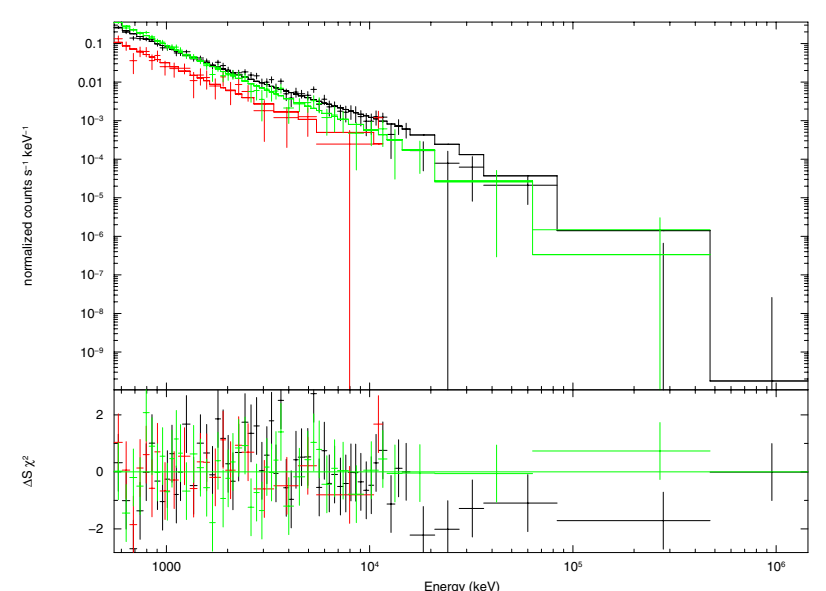

Fig. 4. Superposition of the MCAL spectra in the three time intervals defined in Table 1: $t_{0}-t_{0}+40 \mathrm{~s}$ (interval $\mathrm{A}$, black in colour version), $t_{0}+40 \mathrm{~s}-t_{0}+57 \mathrm{~s}$ (interval $\mathrm{B}$, red in colour version) and $t_{0}+57 \mathrm{~s}-$ $t_{0}+90 \mathrm{~s}$ (interval $\mathrm{C}$, green in colour version).

\subsection{Time-resolved spectral analysis}

The time-integrated spectrum of MCAL between $t_{0}$ and $t_{0}+90 \mathrm{~s}$ can be fit in the energy range $0.5-80 \mathrm{MeV}$ with a single powerlaw and the resulting photon index is $-2.13_{-0.04}^{+0.05}$ with reduced $\chi^{2}=0.92$ and 61 degrees of freedom (d.o.f.). The estimated fluence in the same energy and time intervals is $(2.3 \pm 0.2) \times$ $10^{-4} \mathrm{erg} \mathrm{cm}^{-2}$. All reported errors are at the $90 \%$ confidence level.

We identified three time intervals to study the time-resolved spectrum of the GRB prompt emission, listed in Table 1: the first main bump (from $t_{0}$ until $t_{0}+40 \mathrm{~s}$, hereafter interval $\mathrm{A}$ ), the interbump region (from $t_{0}+40 \mathrm{~s}$ until $t_{0}+57 \mathrm{~s}$, hereafter interval B), and the second main bump (from $t_{0}+57 \mathrm{~s}$ until $t_{0}+90 \mathrm{~s}$, hereafter interval C). We fitted the MCAL spectra of the three intervals in the energy range between 0.5 and $100 \mathrm{MeV}$ with a simple powerlaw and the results are reported in Table 1 . We find evident spectral evolution at a significance level of $4.0 \sigma$, with the first bump harder than the second one (see Fig. 4), as qualitatively expected from the comparison of the bump amplitude and integrated flux in the lightcurves at different energies (in Fig. 1). The first bump is also harder than the interbump region, but in this case a significance level lower than $1 \sigma$ is found, owing to the lower statistics in the spectrum.

Fitting the time integrated spectrum of the GRB data only in GRID with a powerlaw we obtain a photon index of $-2.04_{-0.14}^{+0.31}$, with a total fluence between $22 \mathrm{MeV}$ and $3.5 \mathrm{GeV}$ of $0.25 \pm$ $0.05 \mathrm{ph} \mathrm{cm}^{-2}$, corresponding to $(4.7 \pm 0.9) \times 10^{-5} \mathrm{erg} \mathrm{cm}^{-2}$. We also fitted the joint spectrum of MCAL and GRID with the least squares method using a program specifically developed for the GRID (see for example Pucella et al. 2008). With the available statistics we could only arrange the GRID data in three energy bands (22-70 MeV, 70-200 MeV, and 200-3500 MeV). The joint fit confirms that a single powerlaw, with the same photon index of -2.13 measured in the MCAL time-integrated spectrum, is an adequate model for the spectrum from $500 \mathrm{keV}$ up to $3.5 \mathrm{GeV}$.

GRB 100724B turns out to be the brightest GRB detected by AGILE in gamma rays during its operations in space. The statistics of the GRID data are not good enough to appreciate a possible spectral evolution in the $\mathrm{GeV}$ energy band. We verified the possibility of detecting the same spectral evolution measured by MCAL with the GRID by estimating the significance of a variation in the hardness ratio. Whenever we imposed the same values of the photon indices as measured by MCAL, -2.01 in the first time interval and -2.35 in the third one, and we compared the variation in the resulting hardness ratio with the uncertainty from our data, we find that the variation is at a significance level of $1.3 \sigma$, thus we cannot draw any serious conclusion.

\subsection{Minimum Lorentz factor}

The mere fact that the spectrum of a GRB does not show any cutoff in the GeV energy interval can be used to estimate the minimum Lorentz factor $\Gamma_{\min }$ of the prompt emission fireball because the optical depth of the interactions of gamma rays on low-energy photons is small (Baring \& Harding 1997; Lithwick \& Sari 2001).

We estimated the minimum value of the bulk Lorentz factor for GRB 100724B following the formulae reported in the supporting online material of the paper by Abdo et al. (2009b). The GRID detected a photon of energy as high as $3.5 \pm 1.7 \mathrm{GeV}$ from GRB $100724 \mathrm{~B}$ on $t_{0}+65.9 \mathrm{~s}$. In the estimation of the minimum Lorentz factor, Ackermann et al. (2010b) assume the full width at half maximum $(F W H M)$ of the shortest time pulse as the variability timescale of the emission. Near the arrival time of the highest energy photon $\left(t_{0}+65.9 \mathrm{~s}\right)$, the MCAL lightcurve shows a peak with $F W H M$ of $1.2 \pm 0.1 \mathrm{~s}$, which we assume as the timescale of the burst variability. Since GRB 100724B lacks a redshift, we estimate $\Gamma_{\min }$ as a function of redshift, in an interval between 0 and 5. Assuming a standard cosmology with $H_{0}=70 \mathrm{~km} \mathrm{~s}^{-1} \mathrm{Mpc}^{-1}, \Omega_{\mathrm{M}}=0.3$, and $\Omega_{\Lambda}=0.7$, we obtain a minimum Lorentz factor ranging between $\simeq 50$ and $\simeq 700$ depending on redshift, as shown in Fig. 5. The uncertainty on $\Gamma_{\text {min }}$ is dominated by the uncertainty on the energy reconstruction by GRID, $\Delta E / E \sim 1$ (Tavani et al. 2008). Other ingredients of the overall uncertainty are the errors on the photon index and variability time scale.

\section{Discussion and conclusions}

\subsection{Fluence}

GRB 100724B is characterised by a high fluence, as measured by AGILE both in hard-X rays, $(2.3 \pm 0.2) \times 10^{-4} \mathrm{erg} \mathrm{cm}^{-2}$ in $0.5-80 \mathrm{MeV}$, and in gamma rays, $(4.7 \pm 0.9) \times 10^{-5} \mathrm{erg} \mathrm{cm}^{-2}$ 


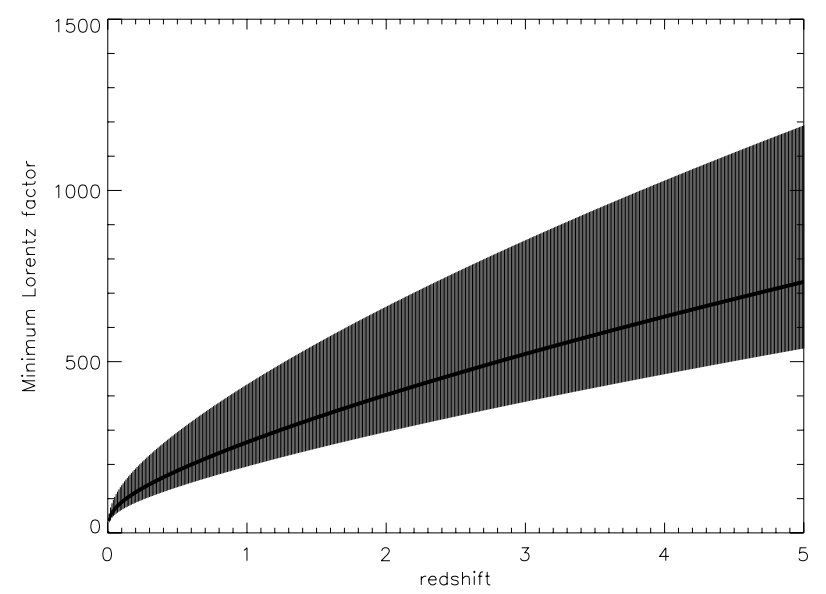

Fig. 5. Estimation of the minimum Lorentz factor, with uncertainty, following the method in the supporting online material of the paper by Abdo et al. (2009b), from the highest energy photon detected by GRID $(3.5 \pm 1.7 \mathrm{GeV})$, the powerlaw photon index $\left(-2.04_{-0.14}^{+0.31}\right)$ and the typical variability timescale of the prompt emission from the MCAL lightcurve $(1.2 \pm 0.1 \mathrm{~s})$.

above $22 \mathrm{MeV}$. We note here that we observed only the initial $\simeq 125 \mathrm{~s}$ of the GRB gamma ray emission, thus we can provide only a lower limit to the fluence in the GRID energy band. Following the analysis by Guiriec et al. (2011) of the GBM observation of GRB 100724B, the burst fluence between $8 \mathrm{keV}$ and $10 \mathrm{MeV}$ is $4.2 \times 10^{-4} \mathrm{erg} \mathrm{cm}^{-2}$, roughly corresponding to $\simeq 2.7 \sigma$ $(\simeq 99.3 \%)$ in the distribution of the fluence values measured by GBM for 121 GRBs (on October 2009) and considered in the analysis by Ghisellini et al. (2010). Rescaling the gamma ray spectrum measured by the AGILE/GRID to the same energy band $(0.1-100 \mathrm{GeV})$ of Ghisellini et al. (2010), we obtain an average energy flux of $5.1 \times 10^{-7} \mathrm{erg} \mathrm{cm}^{-2} \mathrm{~s}^{-1}$, fully consistent with the results of that paper and indicating that GRB 100724B belongs to the same population.

\subsection{Lightcurves and cross-correlation}

While the measures of the burst duration and $T_{90}$ in the hard $\mathrm{X}$-ray band are negligibly affected by the AGILE operating in a spinning mode, thanks to the almost all-sky FoV of the MCAL, we can just put a lower limit at $119 \mathrm{~s}$ on the duration of the $\mathrm{GeV}$ emission since the GRB went out of the GRID FoV at $\simeq t_{0}+125 \mathrm{~s}$ and its emission was fainter than the instrument sensitivity when the burst was back inside, on $t_{0}+410 \mathrm{~s}$ (i.e. $285 \mathrm{~s}$ later). For this reason we cannot draw any serious conclusion about the duration of the GRB in gamma rays from the AGILE data.

The cross-correlation of the lightcurves of GRB 100724B without background subtraction and in various energy ranges (0.3-1 MeV, 1-5 MeV, 5-80 MeV, and $22 \mathrm{MeV}-3.5 \mathrm{GeV}$ ) shows two peculiar characteristics: the simultaneous onset of the gamma ray and hard X-ray emissions and the coincidence of the peak position in the time series. This is demonstrated by the absence of spectral lag between the MCAL lightcurves at different energies, with a bin size of 1.024 s (see Fig. 2) and between the GRID lightcurve and the MCAL ones in three energy ranges, with a bin size of $2.5 \mathrm{~s}$, mainly limited by the statistics of the GRID data (see Fig. 3). Moreover, the first photon is detected by the AGILE/GRID at $10.9 \mathrm{~s}$ after trigger, during the rise of the burst emission in the MeV band. The MCAL and GRID time series remain simultaneous also if we accumulate the data from the whole energy intervals of each instrument, showing that the position of the main bumps is the same, without significant time lags.

The absence of time lag makes GRB 100724B unusual among the events observed so far in gamma rays. In fact, some similarities in the lightcurve can be found in GRB 090217A (Ackermann et al. 2010a), but in that case the first $\simeq 3 \mathrm{~s}$ in the gamma ray lightcurve are considerably fainter than in hard X-rays, and in GRB 080916C (Abdo et al. 2009b), but its gamma ray emission starts at the second peak, $\simeq 3.6 \mathrm{~s}$ after trigger. The lack of gamma rays during the first peak at $\mathrm{MeV}$ of GRB 080916C and the presence of them in the second one and after is used as an argument by Abdo et al. (2009b) to suggest that the two peaks may origin from spatially distinct regions or from two pairs of colliding shells that are different in physical conditions and hardness. Following the same reasons, we can argue that the $\mathrm{GeV}$ and $\mathrm{MeV}$ emissions of GRB 100724B may originate in a common spatial region or, in the internalshock scenario, from the collision of the same pair of shells. Similarly, Maxham et al. (2011) consider the simultaneity of the $\mathrm{GeV}$ and $\mathrm{MeV}$ emission and the single spectral shape across the wide energy band as a more definite argument in favour of the internal shock origin of the gamma ray component, assumed as a spectral extension to lower energies of the $\mathrm{MeV}$ emission.

\subsection{Time resolved spectrum}

GRB 100724B is characterised by an evident spectral evolution in the $\mathrm{MeV}$ energy band. In fact, a significant hard-to-soft variation in the the spectrum from the first $\left(t_{0}-t_{0}+40 \mathrm{~s}\right)$ to the second bump $\left(t_{0}+57 \mathrm{~s}-t_{0}+90 \mathrm{~s}\right)$ is detected by the AGILE/MCAL (see Fig. 4 and Table 1), while the statistics in the gamma ray band does not allow appreciation of a similar evolution. The burst spectral evolution appears stronger if we look at the Konus-Wind results $^{1}$ (Golenetskii et al. 2010) in the $18-1160 \mathrm{keV}$ band, in which the second broad bump $\left(t_{0}+50 \mathrm{~s}-t_{0}+85 \mathrm{~s}\right)$ is brighter than the first one $\left(t_{0}-t_{0}+25 \mathrm{~s}\right)$ and there is also a soft bump, extending approximately from $t_{0}+100 \mathrm{~s}$ until $t_{0}+140 \mathrm{~s}$ and visible only below $70 \mathrm{keV}$ energy, therefore not detected by MCAL. The same feature is also detected by GBM below $200 \mathrm{keV}$ (Guiriec et al. 2011).

The photon index of $-2.13_{-0.04}^{+0.05}$ obtained from the fit of the time-integrated MCAL spectrum (see Table 1) is in good agreement with the highest energy photon index from the Band function measured at $-2.00_{-0.09}^{+0.07}$ by Konus-Wind in the $20 \mathrm{keV}-10 \mathrm{MeV}$ energy range (Golenetskii et al. 2010), although on a longer time interval (from $t_{0}+6 \mathrm{~s}$ to $t_{0}+235 \mathrm{~s}$ ), and with the value of $-1.99 \pm 0.01$ measured by Guiriec et al. (2011) from the Fermi/GBM data. For this burst the peak energy is measured at $369_{-37}^{+42} \mathrm{keV}$ by Konus-Wind (Golenetskii et al. 2010) and at $352 \pm 6 \mathrm{keV}$ by Fermi/GBM Guiriec et al. (2011).

In this analysis of the Fermi/GBM data, Guiriec et al. (2011) find that the fit of the spectra in the $8 \mathrm{keV}-40 \mathrm{MeV}$ energy band significantly improves by including a thermal component of $k T=38.14 \pm 0.87 \mathrm{keV}$. In this case, the high-energy photon index $\beta$ of the Band function is softer, $-2.11 \pm 0.02$. With an energy threshold of $\sim 300 \mathrm{keV}$, the additional spectral component found by Fermi/GBM at $k T=38.14 \pm 0.87 \mathrm{keV}$ cannot be detected by the AGILE/MCAL. Moreover, the reduced chi square value of 0.92 from the MCAL integrated spectrum implies that a simple powerlaw is an adequate model and does not allow us to add other components. Nevertheless, we tried to introduce a thermal component in the spectrum, but it does not affect the

${ }^{1}$ http://wWw.ioffe.ru/LEA/GRBs/GRB100724_T02526/ 
flux above $500 \mathrm{keV}$ and does not improve the reduced chi square (that in fact increases from 0.92 to 0.95 ). For these reasons we can conclude that the MCAL spectum is adequately fit by a simple powerlaw.

The time-resolved spectral analysis by Guiriec et al. (2011) also shows substantial variations in the $E_{\text {peak }}$ parameter of the Band function from $\sim 90 \mathrm{keV}$ up to $\sim 1100 \mathrm{keV}$. In the first half of our interval A, $E_{\text {peak }}$ is higher $(\sim 1100 \mathrm{keV})$ and then decreases down to $\sim 500 \mathrm{keV}$ in the $\mathrm{B}$ and $\mathrm{C}$ time intervals. In the AGILE/MCAL energy range, the spectrum can only be fitted with a powerlaw, thus the $E_{\text {peak }}$ parameter cannot be directly measured. However, the MCAL spectrum is harder (with a lower absolute value of the powerlaw photon index) in the A interval and becomes softer later on, when the absolute value of the photon index increases. The evolution of the photon index in MCAL corresponds to the high-to-low time variation in the $E_{\text {peak }}$ parameter measured by GBM.

Similar to GRB 090902B (Abdo et al. 2009a), the gamma ray emission of GRB 100724B shows large amplitude fluctuations in the $\mathrm{GeV}$ component (of more than a factor of five, see Fig. 1) that can be explained with difficulty in the early afterglow scenario and which are generally attributed to the prompt emission (Abdo et al. 2009a). As in the case of GRB 080916C (Abdo et al. $2009 \mathrm{~b}$ ), the adequacy of a single spectral model from hundreds of $\mathrm{keV}$ up to few $\mathrm{GeV}$ energies is compatible with a non-thermal synchrotron origin of the radiation. The high-energy electrons should, however, upscatter via inverse Compton effect the photons emitted by the synchrotron up to $\mathrm{GeV}$ energies, thus producing a synchrotron self-Compton (SSC) component that should be detected by the AGILE/GRID. The lack of this gamma ray component in the spectrum of GRB 100724B can be explained, as for $080916 \mathrm{C}$, if the magnetic energy density is much higher than the electron density, thus the SSC is suppressed, or if the SSC peaks far above tens of $\mathrm{GeV}$, so cannot be detected by the AGILE/GRID (see the supporting online material of the paper by Abdo et al. 2009b, for further information).

From the cross-correlation analysis and the time-resolved spectral analysis, we find that GRB 100724B is characterised by the presence of a spectral evolution and absence of a spectral lag. These features are rather unusual and are, for example, not found in the other GRBs detected so far in gamma rays by AGILE.

\subsection{Minimum Lorentz factor}

Estimated on the hypothesis of negligible optical thickness at $\mathrm{GeV}$ energy, the minimum Lorentz factor of the GRBs detected in gamma rays ranges between $\simeq 600$ and $\simeq 900$ for the long GRB 080916C (Abdo et al. 2009b) and from $\simeq 700$ to $\simeq 1300$ for the short GRB 090510 (Ackermann et al. 2010b). In the redshift interval from 0 to 5, GRB $100724 \mathrm{~B}$ has a slightly lower minimum Lorenzt factor, between $\simeq 50$ and $\simeq 700$ (see Fig. 5). While the lowest value is similar to the one calculated for GRB 080916C and GRB 090510 for $z=0$, we find only $\simeq 700$ at $z=5$, depending on whether the variability timescale in AGILE is similar to the one measured by Fermi, but the maximum detected photon energy is less due to the lower efficiency of AGILE above $\simeq 1 \mathrm{GeV}$.

Acknowledgements. AGILE is a mission of the Italian Space Agency, with co-participation of INAF (Istituto Nazionale di Astrofisica) and INFN (Istituto Nazionale di Fisica Nucleare). This work was partially supported by ASI grants I/R/045/04, I/089/06/0, I/011/07/0 and by the Italian Ministry of University and
Research (PRIN 2005025417). INAF personnel at ASDC are under ASI contract I/024/05/1. This research has made use of NASA's Astrophysics Data System. Finally, we acknowledge the contribution of the anonymous referee, who stimulated us to substantially improve the quality of the paper.

\section{References}

Abdo, A. A., Ackermann, M., Ajello, M., et al. 2009a, ApJ, 706, L138 Abdo, A. A., Ackermann, M., Arimoto, M., et al. 2009b, Science, 323, 1688 Abdo, A. A., Ackermann, M., Asano, K., et al. 2009c, ApJ, 707, 580 Ackermann, M., Ajello, M., Baldini, L., et al. 2010a, ApJ, 717, L127 Ackermann, M., Asano, K., Atwood, W. B., et al. 2010b, ApJ, 716, 1178 Atwood, W. B., Abdo, A. A., Ackermann, M., et al. 2009, ApJ, 697, 1071 Band, D., Matteson, J., Ford, L., et al. 1993, ApJ, 413, 281

Baring, M. G., \& Harding, A. K. 1997, ApJ, 491, 663

Bevington, P. R., \& Robinson, D. K. 2003, Data reduction and error analysis for the physical sciences (McGraw-Hill)

Bhat, N. 2010, GRB Coordinates Network, Circular Service, 10977

Dingus, B. L. 1995, Ap\&SS, 231, 187

Feroci, M., Costa, E., Soffitta, P., et al. 2007, Nucl. Inst. Meth. Phys. Res. A, 581,728

Fishman, G. J., Meegan, C. A., Wilson, R. B., Paciesas, W. S., \& Pendleton, G. N. 1992, in NASA Conference Publication, ed. C. R. Shrader, N. Gehrels, \& B. Dennis, 3137, 26

Fuschino, F., Labanti, C., Galli, M., et al. 2008, Nucl. Inst. Meth. Phys. Res. A, 588, 17

Gehrels, N., Chincarini, G., Giommi, P., et al. 2004, ApJ, 611, 1005

Gehrels, N., Ramirez-Ruiz, E., \& Fox, D. B. 2009, ARA\&A, 47, 567

Ghisellini, G., Ghirlanda, G., Nava, L., \& Celotti, A. 2010, MNRAS, 403, 926

Giuliani, A. 2010, GRB Coordinates Network, Circular Service, 10996

Giuliani, A., Mereghetti, S., Fornari, F., et al. 2008, A\&A, 491, L25

Giuliani, A., Fuschino, F., Vianello, G., et al. 2010, ApJ, 708, L84

Golenetskii, S., Aptekar, R., Frederiks, D., et al. 2010, GRB Coordinates Network, Circular Service, 10981

Guiriec, S., Connaughton, V., Briggs, M. S., et al. 2011, ApJ, 727, L33

Hascoët, R., Vennin, V., Daigne, F., \& Mochkovitch, R. 2011 [arXiv: 1101.3889]

Hurley, K., Dingus, B. L., Mukherjee, R., et al. 1994, Nature, 372, 652

Kanbach, G., Bertsch, D. L., Fichtel, C. E., et al. 1988, Space Sci. Rev., 49, 69

Kaneko, Y., González, M. M., Preece, R. D., Dingus, B. L., \& Briggs, M. S. 2008, ApJ, 677, 1168

Koshut, T. M., Paciesas, W. S., Kouveliotou, C., et al. 1996, ApJ, 463, 570

Kouveliotou, C., Preece, R., Bhat, N., et al. 1994, ApJ, 422, L59

Kumar, P., \& Barniol Duran, R. 2010, MNRAS, 409, 226

Labanti, C., Marisaldi, M., Fuschino, F., et al. 2009, Nucl. Inst. Meth. Phys. Res. A, 598, 470

Lithwick, Y., \& Sari, R. 2001, ApJ, 555, 540

Marisaldi, M., Fuschino, F., Labanti, C., et al. 2010, GRB Coordinates Network, Circular Service, 10994

Maxham, A., Zhang, B.-B., \& Zhang, B. 2011, MNRAS, 415, 77

Meegan, C., Lichti, G., Bhat, P. N., et al. 2009, ApJ, 702, 791

Mészáros, P. 2006, Rep. Progr. Phys., 69, 2259

Michelson, P. F., Atwood, W. B., \& Ritz, S. 2010, Reports on Progress in Physics, 73, 074901

Moretti, E., Longo, F., Olivo, M., et al. 2009, in Società Italiana di Fisica Conference Proceedings, The Shocking Universe - Gamma Ray Bursts and High Energy Shock Phenomena, 102

Norris, J. P. 2002, ApJ, 579, 386

Piran, T. 2004, Rev. Mod. Phys., 76, 1143

Preece, R. D., Briggs, M. S., Mallozzi, R. S., et al. 2000, ApJS, 126, 19

Prest, M., Barbiellini, G., Bordignon, G., et al. 2003, Nucl. Inst. Meth. Phys. Res. A, 501, 280

Pucella, G., Vittorini, V., D’Ammando, F., et al. 2008, A\&A, 491, L21

Rossi, A., Schulze, S., Klose, S., et al. 2011, A\&A, 529, A142

Schaefer, B. E., Palmer, D., Dingus, B. L., et al. 1998, ApJ, 492, 696

Schneid, E. J., Bertsch, D. L., Fichtel, C. E., et al. 1992, A\&A, 255, L13

Schneid, E. J., Bertsch, D. L., Dingus, B. L., et al. 1995, ApJ, 453, 95

Sommer, M., Bertsch, D. L., Dingus, B. L., et al. 1994, ApJ, 422, L63

Tanaka, Y., Ohno, M., Takahashi, H., et al. 2010, GRB Coordinates Network, Circular Service, 10978, 10978

Tavani, M., Barbiellini, G., Argan, A., et al. 2008, Nucl. Inst. Meth. Phys. Res. A, 588,52

Tavani, M., Barbiellini, G., Argan, A., et al. 2009, A\&A, 502, 995

Zhang, B. 2011, C. R. Phys., 12, 206 